\title{
Resource Allocation for Cognitive Satellite Communications with Incumbent Terrestrial Networks
}

\author{
Eva Lagunas*, Member, IEEE, Shree Krishna Sharma, Member, IEEE, Sina Maleki, Member, IEEE, Symeon \\ Chatzinotas, Senior Member, IEEE, Björn Ottersten, Fellow, IEEE
}

\begin{abstract}
The lack of available unlicensed spectrum together with the increasing spectrum demand by multimedia applications has resulted in a spectrum scarcity problem, which affects Satellite Communications (SatCom) as well as terrestrial systems. The goal of this paper is to propose Resource Allocation (RA) techniques, i.e. carrier, power and bandwidth allocation, for a cognitive spectrum utilization scenario where the satellite system aims at exploiting the spectrum allocated to terrestrial networks as the incumbent users without imposing harmful interference to them. In particular, we focus on the microwave frequency bands $17.7-19.7 \mathbf{G H z}$ for the cognitive satellite downlink and $27.5-29.5 \mathbf{G H z}$ for the cognitive satellite uplink, although the proposed techniques can be easily extended to other bands. In the first case, assuming that the satellite terminals are equipped with multiple Low Block Noise Converters (LNB), we propose a joint beamforming and carrier allocation scheme to enable cognitive Space-to-Earth communications in the shared spectrum where Fixed Service (FS) microwave links have priority of operation. In the second case, however, the cognitive satellite uplink should not cause harmful interference to the incumbent FS system. For the latter, we propose a Joint Power and Carrier Allocation (JPCA) strategy followed by a bandwidth allocation scheme which guarantees protection of the terrestrial FS system while maximizing the satellite total throughput. The proposed cognitive satellite exploitation techniques are validated with numerical simulations considering realistic system parameters. It is shown that the proposed cognitive exploitation framework represents a promising approach for enhancing the throughput of conventional satellite systems.
\end{abstract}

Index Terms-Resource Allocation, Cognitive Radio, Satellite Communications, Carrier Allocation, Beamforming.

\section{INTRODUCTION}

$\mathbf{S}$ ATELLITE communications (SatCom) [1]-[3] is a natural outgrowth of modern technology and of the continuing demand for greater capacity, higher quality in communications and wider coverage. Satellite technology is ideally suited to

The authors are with the Interdisciplinary Centre for Security, Reliability and Trust (SnT), University of Luxembourg (http://www.securityandtrust.lu), L-2721 Luxembourg. E-mail: \{eva.lagunas, shree.sharma, sina.maleki, symeon.chatzinotas, bjorn.ottersten\}@uni.lu

The research leading to these results has received funding from the the European Commission in the framework of the FP7 CoRaSat (Grant agreement no. 316779), from the European Union's Horizon 2020 research and innovation programme in the framework of SANSA (Grant agreement no. 645047) and by the National Research Fund, Luxembourg, under CORE project SeMIGod, and CORE project SATSENT.

Part of this work was presented to the IEEE International Conference on Communications (ICC) 2015 and to the International Conference on Cognitive Radio Oriented Wireless Networks (CROWNCOM), 2015.

* Corresponding author. deploy a network that has wide coverage since it is able to overcome long distances and inhospitable terrains, and can be rapidly put in place. For the most remote and sparsely populated locations, broadband access could practically be offered by a satellite, which is likely to be the cheapest broadband solution in these cases.

Satellites have been successfully serving the traditional markets, i.e., telephony and broadcasting, but more recently and led by the penetration increase of internet on human life, satellites are more and more used as a broadband access solution. The demand for broadband satellite services is growing at unprecedented rates and the licensed spectrum of $500 \mathrm{MHz}$ for exclusive use, both for uplink and downlink, in the Ka band has been shown to be insufficient to meet the forthcoming demands [4], [5].

To enhance the spectral efficiency, satellite systems have moved from single beam and $\mathrm{C} / \mathrm{Ku}$ band to multi-beam and $\mathrm{Ka}$ band satellites [6], [7]. However, the maximum system capacity of current multi-beam satellites is limited by the fractional frequency reuse. Aggressive frequency reuse schemes have been shown to be promising towards enhancing the spectral efficiency of SatCom [8]. Excessive co-channel interference is the main limitation of the high frequency reuse systems, which, on their own, fall short of meeting the increasing data rate demand. In this regard, the concept of Cognitive Radio (CR) technology has emerged as a promising solution to enhance the satellite spectrum utilization by enabling dynamic spectrum access between two satellite systems [9], [10] or between satellite and terrestrial systems [11], [12].

The main functions of a CR network are spectrum awareness and spectrum exploitation [13]. Spectrum awareness is the function in charge of obtaining relevant information of the surrounding radio environment, including the level of exploitation of the radio frequency resources. In this context, interference estimation techniques [14], [15] and the determination of cognitive zones [16]-[18] (geographical areas where CR techniques should be apply to manage interference) have been investigated in the context of cognitive SatCom. Spectrum exploitation, which receives as input the spectrum awareness information, becomes an essential capability for CRs since it is responsible to optimally distribute the available resources within the secondary devices. This should be done in a way that the secondary network throughput is maximized and the licensed network is guaranteed not to suffer harmful interferences coming from the cognitive transmitters. Here, 


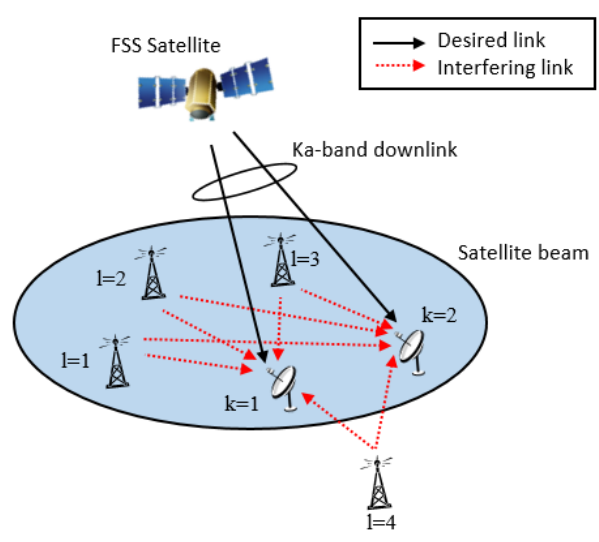

(a)

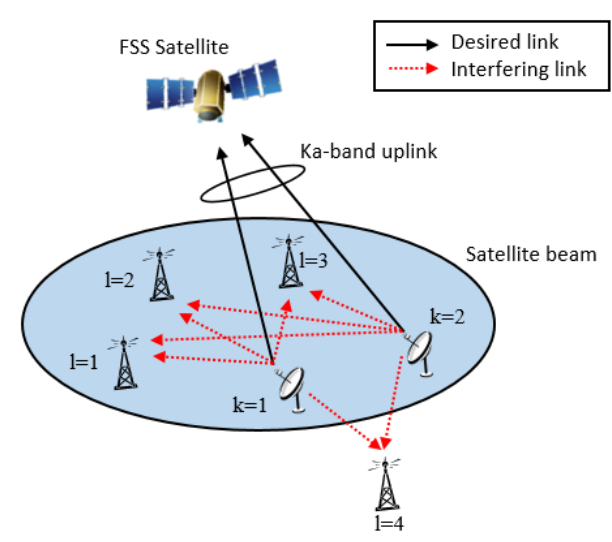

(b)

Fig. 1. Spectral co-existence of FSS with the FS terrestrial links in Ka-band: (a) FSS downlink in 17.7-19.7 GHz band, (b) FSS uplink in 27.5-29.5 GHz band.

we develop the required techniques for spectrum exploitation assuming that the spectrum awareness is achieved through other means, e.g., databases. Most CR research related to resource management has focused on the terrestrial part [19], while only a few contributions study this problem in the cognitive satellite framework [20], [21].

In this paper, we provide a description of the techniques adopted for spectrum exploitation in two of the scenarios discussed in [12], [22]: (i) the cognitive satellite downlink in the $17.7-19.7 \mathrm{GHz}$ band, and (ii) the cognitive satellite uplink in the $27.5-29.5 \mathrm{GHz}$ band. These scenarios have been chosen based on market, business and technical feasibility analysis [23]. In the $17.7-19.7 \mathrm{GHz}$ band, the FS links are incumbent links, but uncoordinated Fixed Satellite Service (FSS) terminals can also be deployed without right of protection. The term "incumbent" in this paper refers to the (licensed) system who has higher priority or legacy rights on the usage of a specific part of the spectrum. On the other hand, the $27.5-29.5 \mathrm{GHz}$ band entails the FSS terminal operating in uplink and thus is a potential interferer to the incumbent FS links operating in that spectrum. In the latter scenario, the FSS system should limit the potential interference generated towards incumbent FS links. These two scenarios and the related existing resource management works are detailed later in Section II. Our goal in this paper is to exploit the spectrum opportunities in such a way that the cognitive satellite system throughput is maximized and the cognitive activity is kept unnoticed by the incumbent terrestrial system. Nevertheless, it is worth to mention that, although we consider the two aforementioned scenarios as use cases, the proposed techniques can be easily extended to any similar cognitive satellite applications. The proposed techniques are evaluated through numerical simulations using realistic system parameters.

This paper is organized as follows. Section II describes the scenarios under consideration and presents a review of the most prominent published related works. Section III introduces the signal and interference model. The cognitive exploitation mechanisms for both the downlink and the uplink scenario are formalized mathematically in Section IV. Section V provides supporting results based on numerical data. Finally, Section VI concludes the paper.

Notation: Throughout the paper, scalars are denoted by nonboldface type, vectors by boldface lowercase letters and matrices by boldface uppercase letters. Superscripts $(\cdot)^{T},(\cdot)^{*}$ and $(\cdot)^{H}$ denote transpose, complex conjugate and complex conjugate transpose, respectively. Let $\|\mathbf{a}\|_{l_{p}}$ denote the $l_{p}$-norm of vector a, i.e., $\|\mathbf{a}\|_{l_{p}}=\left(\sum_{i=1}^{n}\left|a_{i}\right|^{p}\right)^{1 / p}$.

\section{Scenario Definition, Related Work AND CONTRIBUTIONS}

In this section, a short description of the two considered cognitive satellite scenarios are presented together with a short review of the regulatory context that applies to the frequency bands under consideration. We also review the most prominent works related to resource allocation in cognitive SatCom and highlight the contributions of this paper.

\section{A. Cognitive Satellite Downlink}

In this section, we consider the cognitive downlink access by Geostationary (GEO) FSS terminals in the band 17.7$19.7 \mathrm{GHz}$, where the incumbent users are Fixed-Service (FS) microwave links. Fig. 1(a) depicts the considered scenario.

Within Europe, the European Conference of Postal and Telecommunications Administrations (CEPT) has adopted Decision ECC/DEC/(00)07 [24] to allow uncoordinated FSS terminals to co-exist with FS links in the $17.7-19.7 \mathrm{GHz}$ band but without right of protection. In this case, the downlink interference from the cognitive satellite to the terrestrial FS receivers is negligible due to the limitation in the maximum EIRP density of the current Ka band satellite system [25]. However, the interference from FS transmitters to the cognitive satellite terminal needs to be taken into account in order to guarantee operation of the cognitive users.

Several resource management strategies for non-cognitive satellite downlink have been investigated in the literature 
[26]-[29]. In [26], the authors consider the whole multibeam satellite system design and they propose to allocate different bandwidth and power to each beam according to the asymmetrical traffic demand among the beams. In [27], the issue of multi-beam power allocation is solved considering both traffic demands and channel conditions over satellite downlinks. Carrier frequency assignment for military SatCom in which balance between spectral efficiency and resilience is taken into account was presented in [28]. In [29], it is shown that the overall satellite performance can be improved when resource allocation is done considering co-operation of several protocol layers.

Nevertheless, research works referring to resource allocation for cognitive satellite downlink are rather limited [20], [30], [31]. In [30], the authors propose an optimization of the frequency reuse and polarization (often referred as color) as a first coarse radio resource allocation in order to minimize the interference received by incumbent FS stations. Here, we go a step further and, building on our work in [20], we consider an efficient receive beamforming technique combined with optimal carrier allocation in order to maximize the overall downlink throughput. More specifically, a single beam evaluation was carried out in [20] with a simple free space path loss model. Recently, the diffraction effect based on the terrain data has been identified as an extremely significant component in the interference modeling [32]. Here, unlike [20], we provide a more complete study by considering multiple beams and considering the diffraction loss on top of the free space path loss. The power allocation in this scenario is assumed to be controlled by the Adaptive Coding and Modulation (ACM) capabilities of the DVB-S2X standard [33]. Similar joint beamforming and carrier allocation problem has been studied in [31] for enabling the spectral coexistence of GEO FSS downlink with the Broadcasting Satellite Services (BSS) feeder links in $17.3-18.1 \mathrm{GHz}$ band.

In this scenario, we assume the FSS terminal to be equipped with multiple Low Block Noise Converter (LNB) based on Feed Array Reflector (FAR). According to [34], the cost of a consumer grade single LNB is low and the compact design of multiple LNBs using dielectric feed elements is feasible. However, the number of LNBs should be kept low, e.g., 2-3 LNBs, due to cost, mechanical support and electromagnetic blockage issues, which limits the degrees of freedom of the beamforming design.

\section{B. Cognitive Satellite Uplink}

In this section, we consider the cognitive Geostationary Orbit (GEO) satellite uplink where satellite terminals reuse frequency bands of FS terrestrial microwave links which are the incumbent users in the Ka 27.5-29.5 GHz band. Fig. 1(b) depicts the considered scenario.

As in the previous case, ITU-R assigns FS links as the incumbent users with the highest priority in this band, while keeping the FSS terminals as the co-incumbent with FS links having the right of protection from the FSS terminals. Particularly, the shared civil Ka uplink bands in Europe are ruled by the ECC Decision (05)01 [35]. Essentially, CEPT sets the band 29.5-30 GHz for exclusive FSS use (same as ITU-R) and sets the conditions under which $27.5-29.5 \mathrm{GHz}$ spectrum can be used by uncoordinated earth stations (i.e., broadband terminals) while not interfering with the FS links. In this paper, we investigate FSS cognitive satellite terminals operating in 27.5-29.5 GHz, dynamically sharing frequency spectrum of FS microwave links with priority protection. Consequently, cognitive satellite uplink communication is not performed unless the interference caused at the incumbent system is below a pre-defined threshold. This scenario falls within the underlay CR paradigm [19], where the terrestrial system is licensed to freely exploit the spectrum; whereas cognitive satellite system is allowed to utilize the same spectrum as long as it does not affect the licensed communication. In general, the maximum interference level that the FS microwave system is willing to tolerate is defined by the regulatory authorities. To protect FS receivers from FSS satellite transmissions, the ITU Radio Regulations impose constraints on the FSS terminal transmission power so that they operate below the noise floor of the incumbent users. Here, we consider only the long term interference criteria which is typically taken as 6 or $10 \mathrm{~dB}$ below the FS receiver noise [36], [37].

According to DVB-RCS [38], the Network Control Center (NCC) of the satellite system is the entity that distributes the available resources according to the collected traffic demands of the FSS terminals (in the return link, the FSS terminals are usually known as Return Channel Satellite Terminal (RCST). As in the forward link, the available literature on resource allocation for the return link is mainly related to non-cognitive satellite systems [39]-[41]. In [39], timeslots are assigned according to users' demands and dynamically taking into account the variations of the propagation conditions. In [40], network congestion is solved by performing an optimized resource allocation considering a cross-layer interaction. A cross-layer framework for optimizing the resource allocation of a satellite return link is proposed in [41], where Medium Access Control (MAC) methods are designed taking into account the adaptive physical layer.

The applicability of $\mathrm{CR}$ in the aforementioned scenario was discussed in [42], concluding that both satellite and terrestrial systems could potentially operate in the same band without degrading each others' performance. Of particular interest for the present work is [43], where the same cognitive satellite uplink paradigm was considered. Specifically, [43] proposes an interference-based constraint on the inverse Signal-toInterference plus Noise Ratio (iSINR). However, [43] neglects the aggregate interference caused by multiple RCSTs. It is important to note that, although a Multi-Frequency Time Division Multiple Access (MF-TDMA) scheme is employed in the DVB-RCS2 standard for the return link [38], it may happen that more than one RCST while operating on different carrier frequencies produce aggregated interference to the FS microwave network because the carrier bandwidth of the FS microwave links is usually higher than that of the RCSTs [44]. Here, we take this into account and propose a simple and efficient joint power and carrier allocation (JPCA) technique for the cognitive satellite uplink and terrestrial FS co-existence scenario followed by a bandwidth allocation scheme that 
allocates bandwidth according to the user rate demands. This is an extension of our work in [21], where the bandwidth per user was considered fixed and the cognitive satellite uplink scenario was evaluated considering an "artificial" beam (generated with MATLAB) and with a simple free space propagation loss. Here, we add the bandwidth resource to the optimization problem and provide a more complete study by considering realistic multiple beams provided by a satellite manufacturer and taking the diffraction loss into account.

\section{Signal And Interference Model}

This section presents the signal and interference model between the cognitive satellite system and the FS microwave links under the considered scenarios.

Let us assume a scenario with $K$ FSS terminals and $L$ FS microwave stations and $M$ carrier frequencies available at the satellite. Throughout the paper, we assume $K=M$ which means that only one FSS terminal is assigned per carrier. If $K>M$, Time Division Multiple Access (TDMA) is considered in DVB-S2(X) on the forward link and MultiFrequency Time Division Multiple Access (MF-TDMA) is considered in DVB-RCS on the return link, which allows several users to share the same carrier frequency by dividing the signal into different time/frequency slots. In this case, the optimal scheduling problem should be considered, which is the subject of future work.

\section{A. Cognitive Satellite Downlink}

Following similar notations as in [20], the received signal level at the $k$-th FSS terminal can be expressed as

$$
P_{\mathrm{Rx}}(k)=P_{\mathrm{Tx}}^{\mathrm{SAT}} \cdot G_{\mathrm{Tx}}^{\mathrm{SAT}}(k) \cdot G_{\mathrm{Rx}}^{\mathrm{FSS}}(0) \cdot L_{s},
$$

where,

- $P_{\mathrm{Tx}}^{\mathrm{SAT}}$ : Transmit power of the FSS satellite.

- $G_{\mathrm{Tx}}^{\mathrm{SAT}}(k)$ : Transmit satellite beam gain for the $k$-th FSS terminal user.

- $G_{\mathrm{Rx}}^{\mathrm{FSS}}(0)$ : Gain of the FSS terminal antenna at the boresight angle. The radiation pattern can be obtained from ITU-R S.465-6 [45].

- $L_{s}$ : Free space path loss computed as $\left(\frac{c}{4 \pi d f}\right)^{2}$, where $c$ is the propagation speed, $f$ the frequency and $d$ the distance. The distance between the FSS terminal and the satellite is assumed to be $35,786 \mathrm{~km}$.

In the downlink scenario considered in this paper, the cognitive satellite does not impose interference to the incumbent terrestrial system due to the regulatory constraints on the power flux density of the satellite on the surface of the earth. However, the FSS terminals may experience interference from the FS links.

Assuming that the $k$-th FSS terminal is operating on a particular carrier frequency $f_{m}$, it will receive interference from the corresponding FS microwave stations working on the same $f_{m}$. Let us denote $I_{k}(l, m)$ as the interference level caused by a single $l$-th FS terminal at the $m$-th carrier at the $k$-th FSS terminal. The latter can be written as,

$$
I_{k}(l, m)=P_{\mathrm{Tx}}^{F S}(l) \cdot G_{\mathrm{Tx}}^{F S}\left(\theta_{l, k}\right) \cdot G_{\mathrm{Rx}}^{\mathrm{FSS}}\left(\theta_{k, l}\right) \cdot L_{s} \cdot L_{d},
$$

where,

- $P_{\mathrm{Tx}}^{F S}(l)$ : Transmit power of the $l$-th FS station.

- $G_{\mathrm{Tx}}^{F S}(\theta)$ : Gain of the FS transmitting antenna at an offset angle $\theta$. The radiation pattern can be obtained from ITUR F.1245-2 [46].

- $\theta_{i, j}$ : Offset angle (from the boresight direction) of the $i$-th station in the direction of the $j$-th station.

- $G_{\mathrm{Rx}}^{\mathrm{FSS}}(\theta)$ : Gain of the FSS terminal receiving antenna at an offset angle $\theta$. The radiation pattern can be obtained from ITU-R S.465-6 [45].

- $L_{d}$ : Diffraction loss computed according to the Bullington model described in ITU-R P.526-13 "Propagation by diffraction" [47].

As in (1), $L_{s}$ denotes the free space path loss. Note that (2) assumes that the interfering signal falls within the victim bandwidth. If the spectra do not overlap completely, then a compensation factor of $B_{\text {overlap }} / B^{\mathrm{FSS}}$ is applied, where $B_{\text {overlap }}$ stands for the portion of the interfering signal spectral density within the receive modem filter bandwidth given by $B^{\mathrm{FSS}}$.

The aggregated interference from the whole terrestrial system received at the $k$-th FSS terminal for a particular carrier frequency $f_{m}$ is thus given by

$$
I_{k}(m)=\sum_{l=1}^{L} I_{k}(l, m) .
$$

According to (1) and (3), the Signal-to-Interference plus Noise Ratio (SINR) per user and per carrier in the forward link can be computed as follows,

$$
\operatorname{SINR}_{\mathrm{fwd}}(m, k)=\frac{P_{\mathrm{Rx}}(k)}{I_{k}(m)+I_{\mathrm{fwd}}^{\mathrm{co}}+N_{0}},
$$

where $I_{\text {fwd }}^{\text {co }}$ is the co-channel interference in the forward link caused due the frequency re-use in multi-beam satellite forward communications and $N_{0}$ is the noise thermal power. For notation convenience, we stack the individual SINR values in matrix $\mathbf{S I N R}_{\mathrm{fwd}} \in \mathbb{R}^{M \times K}$ as follows,

$$
\mathbf{S I N R}_{\mathrm{fwd}}=\left[\begin{array}{ccc}
\operatorname{SINR}_{\mathrm{fwd}}(1,1) & \cdots & \operatorname{SINR}_{\mathrm{fwd}}(1, K) \\
\vdots & \ddots & \vdots \\
\operatorname{SINR}_{\mathrm{fwd}}(M, 1) & \cdots & \operatorname{SINR}_{\mathrm{fwd}}(M, K)
\end{array}\right],
$$

where the rows indicate the carrier frequencies and the columns indicate the FSS terminal users.

\section{B. Cognitive Satellite Uplink}

The cognitive satellite uplink, on the other hand, entails the FSS terminal transmitting from Earth-to-Space and thus is a potential interferer to the incumbent FS links operating in the $28 \mathrm{GHz}$ region, as illustrated in Fig. 1(b). For the purpose of protecting FS terrestrial links and following the notation introduced in [21], the following interference constraints must be satisfied,

$$
I_{l}(m) \leq I_{\mathrm{thr}, l} \quad \text { for } \quad l=1, \ldots, L,
$$

where $I_{\mathrm{thr}, l}$ denotes the tolerable interference level at the $l$ th FS receiver and $I_{l}(m)$ denotes the aggregated interference power caused by all $K$ cognitive transmitters at the $l$-th FS 


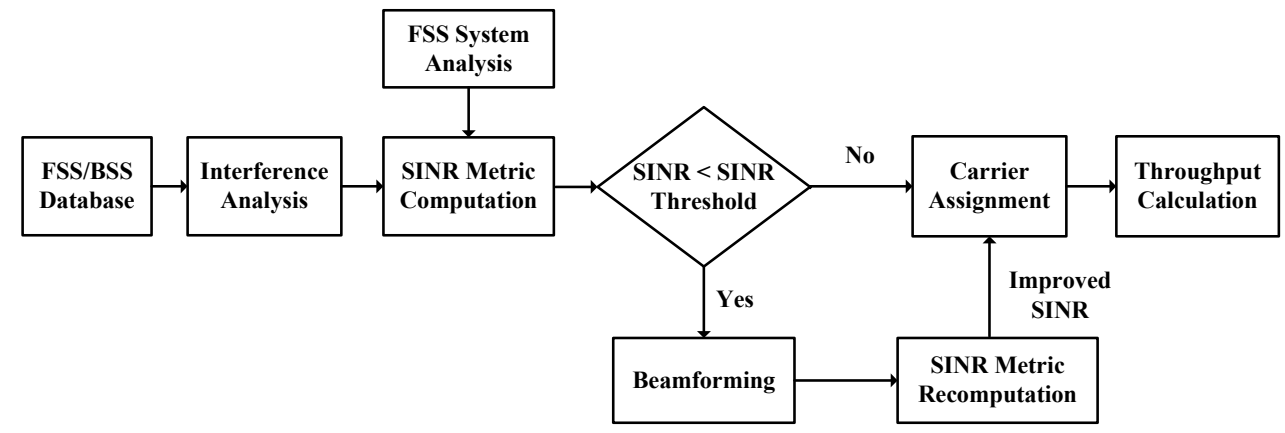

Fig. 2. Block diagram of the cognitive exploitation framework.

microwave station for a particular carrier frequency $f_{m}$. The latter can be expressed as follows,

$$
I_{l}(m)=\sum_{k=1}^{K} p(m, k) \cdot g_{k, l}(m),
$$

where $p(m, k)$ denotes the transmit power of the $k$-th FSS terminal at the $m$-th carrier and $g_{k, l}(m)$ being the instantaneous cross-channel link gain from the $k$-th FSS terminal to the $l$-th FS station for the transmitting frequency $f_{m}$, which is given by,

$$
g_{k, l}(m)=G_{\mathrm{Tx}}^{F S S}\left(\theta_{k, l}\right) \cdot G_{\mathrm{Rx}}^{\mathrm{FS}}\left(\theta_{l, k}\right) \cdot L_{s} \cdot L_{d},
$$

where,

- $G_{\mathrm{Tx}}^{F S S}(\theta)$ : Gain of the RCST transmitting antenna at offset angle $\theta$. The radiation pattern can be obtained from ITU-R S.465-6 [45].

- $G_{\mathrm{Rx}}^{\mathrm{FS}}(\theta)$ : Gain of the FS receiving antenna at offset angle $\theta$. The radiation pattern can be obtained from ITU-R F.1245-2 [46].

As in (1) and (2), $L_{s}$ denotes the free space path loss. Again, if the spectra do not overlap completely, then a compensation factor of $B_{\text {overlap }} / B^{\mathrm{FS}}$ should be applied, where, $B_{\text {overlap }}$ stands for the portion of the interfering signal spectral density within the receive filter bandwidth given by $B^{\mathrm{FS}}$.

Throughout this paper, we assume the availability of a FS database from which the FS antenna location and pointing directions can be perfectly known. This information is used for the proper estimation of $g_{k, l}(m)$. Clearly, the accuracy and completeness of the available database determines the quality of the estimation. In this respect, investigation of techniques to improve the robustness of the database information will be considered in future works.

For notational convenience, we stack the individual channel gain values in matrix $\mathbf{G}(m) \in \mathbb{R}^{K \times L}$ as follows,

$$
\mathbf{G}(m)=\left[\begin{array}{ccc}
g_{1,1}(m) & \cdots & g_{1, L}(m) \\
\vdots & \ddots & \vdots \\
g_{K, 1}(m) & \cdots & g_{K, L}(m)
\end{array}\right] .
$$

Moreover, each RCST have its own power limits dictated by the CEPT regulation, which states that the maximum EIRP of RCSTs shall not exceed a value in a range from $55 \mathrm{dBW}$ to $60 \mathrm{dBW}$ [48]. This limitation will be denoted in this paper by $P_{k}^{\max }, k=1, \ldots, K$. Therefore, $p(m, k) \leq P_{k}^{\max }, \forall k$.
Finally, the SINR level per carrier and per user in the return link can be derived as follows,

$$
\operatorname{SINR}_{\mathrm{rtn}}(m, k)=\frac{p(m, k) \cdot G_{\mathrm{Tx}}^{\mathrm{FSS}}(0) \cdot G_{\mathrm{Rx}}^{\mathrm{SAT}}(k) \cdot L_{s}}{I_{\mathrm{rtn}}^{\mathrm{co}}+N_{0}},
$$

where $G_{\mathrm{Tx}}^{\mathrm{FSS}}(0)$ is the boresight RCST antenna gain, $G_{\mathrm{Rx}}^{\mathrm{SAT}}(k)$ is the receive satellite beam gain for the $k$-th FSS terminal user and $I_{\mathrm{rtn}}^{\mathrm{co}}$ is the co-channel interference caused due the frequency reuse in multi-beam return SatCom. Again, the values $\operatorname{SINR}_{\mathrm{rtn}}(m, k)$ can be rearranged in a matrix notation as follows,

$$
\mathbf{S I N R}_{\mathrm{rtn}}=\left[\begin{array}{ccc}
\operatorname{SINR}_{\mathrm{rtn}}(1,1) & \cdots & \operatorname{SINR}_{\mathrm{rtn}}(1, K) \\
\vdots & \ddots & \vdots \\
\operatorname{SINR}_{\mathrm{rtn}}(M, 1) & \cdots & \operatorname{SINR}_{\mathrm{rtn}}(M, K)
\end{array}\right],
$$

where the rows indicate the carrier frequencies and the columns indicate the RCSTs.

\section{Cognitive Spectrum Exploitation Framework}

One of the major challenges for cognitive SatCom is how to design efficient resource allocation algorithms so that the overall satellite throughput is maximized and (i) the incumbent FS communication is not disturbed, and (ii) the effect of the incumbent system to the cognitive communication is minimized. This section presents the cognitive spectrum exploitation framework for the two scenarios described in Section III. In particular, an efficient receive beamforming technique combined with carrier allocation is proposed for the cognitive satellite downlink scenario. With regard to the cognitive satellite uplink, the interference constraints at the FS microwave stations limit the transmit power of RCSTs. Therefore, power and frequency resources have to be jointly allocated so as to satisfy interference constraints and to maximize the satellite system performance.

\section{A. Cognitive Satellite Downlink}

For the cognitive satellite downlink, we propose to employ beamforming techniques at the FSS terminal in order to cancel out strong interferences caused by the incumbent FS microwave transmitters. Then, the carrier allocation module receives the SINR for each user over each available carrier as the input and assigns the carriers to the users by maximizing the overall satellite throughput. 
The block diagram of the proposed cognitive exploitation framework is depicted in Fig. 2. First, a geolocation database provides the sufficient information to determine the interference level $I_{k}(m)$ as described in Section III-A. Subsequently, the $\operatorname{SINR}_{\text {fwd }}(m, k)$ values are computed and, only the FSS terminals which suffer excessive interference apply beamforming to improve the SINR. Finally, these improved SINR values are fed to the carrier allocation module in order to allocate the available spectrum resources by maximizing the overall throughput.

1) Beamforming design: In this paper, we use the general Linearly Constrained Minimum Variance (LCMV) beamformer proposed by Frost in 1972 [49]. The LCMV allows the steered beam to focus onto the desired direction (satellite direction) while imposing multiple linear constraints relative to the FS interference directions. The information on the interference directions is a-priori known with the availability of the FS database.

The number of antennas that can be installed at the satellite terminal is limited due to cost and implementation aspects and, thus, the number of nulls that can be created are limited. In general, the number of LNBs should be kept low, e.g., 2-3 LNBs, due to cost, mechanical support and electromagnetic blockage issues [34]. To deal with the limitation of higher number of interference with respect to antennas, we include only the strongest FS interfering link to the beamforming design. The proposed beamforming application in the considered scenario is described with detail in Algorithm 1. In Algorithm 1 , we use $\hat{\mathbf{R}}_{y}$ to denote the sample covariance matrix of the received signal defined as $\hat{\mathbf{R}}_{y}=\frac{1}{N_{s}} \sum_{n=1}^{N_{s}} \mathbf{y}(n) \mathbf{y}^{H}(n)$, where $\mathbf{y}(n)$ and $N_{s}$ denote the received snapshot at a time $n$ and the total number of available snapshots, respectively.

2) Carrier Allocation: This module receives the SINR values after having applied the beamforming phase. Now, it is time to assign the available carrier frequencies among the FSS terminals such that the sum-rate of the cognitive satellite downlink is maximized.

Let $\mathbf{A}=\left[\begin{array}{lll}\mathbf{a}_{1} & \cdots & \mathbf{a}_{K}\end{array}\right]$ be the carrier allocation matrix, where the elements of $\mathbf{a}_{k} \in \mathbb{R}^{M \times 1}$ work as an indicator function: " 1 " if $m$-th carrier is assigned to the $k$-th user and " 0 " otherwise. Therefore, for each carrier $m$, we have $\forall m$ : $\sum_{k=1}^{K} \mathbf{a}_{k}(m)=1$, where $\mathbf{a}_{k}(m)$ denotes the $m$-th component of vector $\mathbf{a}_{k}$.

One of the most popular figure of merit for measuring system performance is the sum-rate. The maximization of the cognitive satellite sum-rate can be expressed as,

$$
\max _{\mathbf{A}}\left\|\operatorname{vec}\left(\mathbf{A} \odot \mathbf{R}\left(\mathbf{S I N R}_{B F}\right)\right)\right\|_{l_{1}} \quad \text { s.t. } \quad \sum_{k=1}^{K} \mathbf{a}_{k}(m)=1,
$$

where $\odot$ denotes the Hadamard product, $\operatorname{vec}(\cdot)$ denotes the vectorization operator, $\|\cdot\|_{l_{1}}$ denotes the $l_{1}$-norm and $\mathbf{R}(\mathbf{S I N R})$ denotes the rate matrix with elements $r(m, k), k=1, \ldots, K$, $m=1, \ldots, M$, e.g. the DVB-S2X rate [33] associated with the corresponding SINR value.

The optimization problem in (12) can be solved using the Hungarian algorithm [50], which provides an efficient and low complexity method to solve the one-to-one assignment

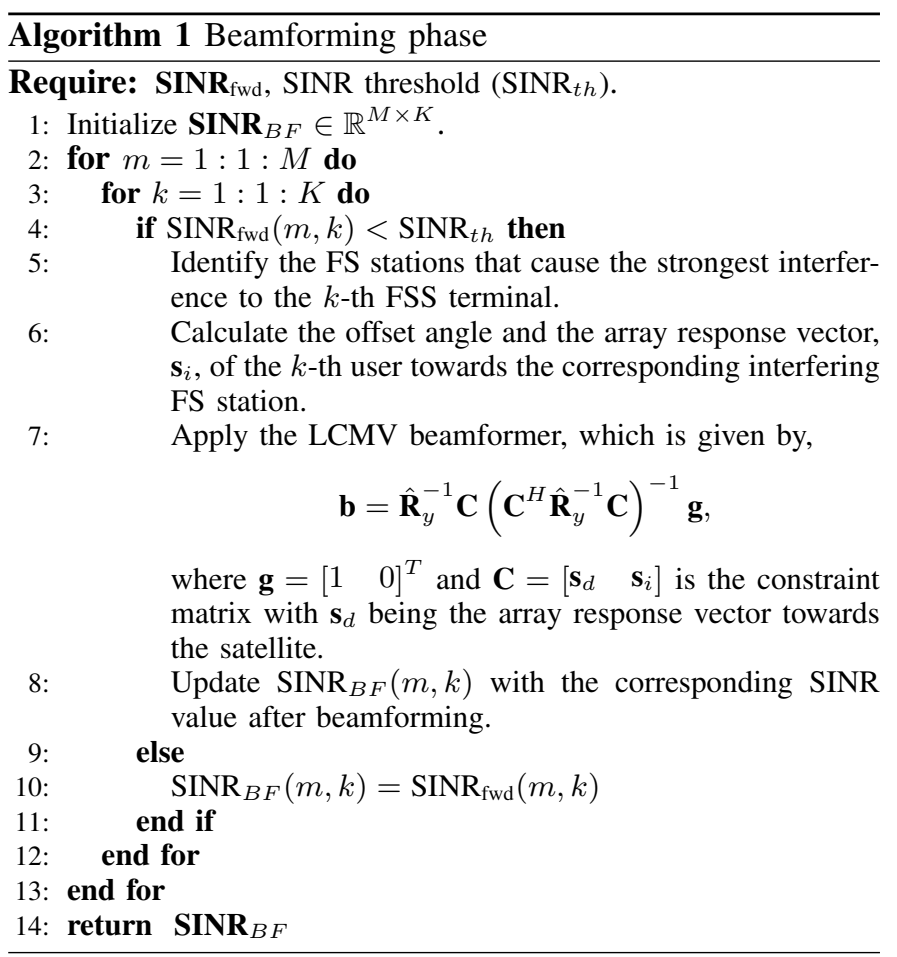

problem in polynomial time.

\section{B. Cognitive Satellite Uplink}

The goal in this scenario is to optimally assign carriers and users' bandwidth, and adjust the transmit power levels $p(m, k)$ so that the satellite system performance is maximized while the aggregated interference caused at the incumbent FS system is kept below the predefined threshold. From (7), it can be observed that all three parameters, i.e. transmitted powers, carriers and bandwidths, are key factors in the total interference seen from the FS stations. Therefore, power, carrier and bandwidth allocation should be considered jointly. However, global optimization of these three resources is an open research problem which is out of the scope of the present paper. Moreover, complex optimization algorithms would result into computationally unaffordable allocation approaches which are not suitable for the near-future satellite systems. Here, we focus on sub-optimal but simple and efficient techniques. In particular, we first focus on the power and carrier allocation such that a maximum interference level received at the FS system is guaranteed. This refers to the JPCA module introduced by the authors in [21]. In a second step, we propose a bandwidth allocation technique based on the minimum rate requested by the RCSTs, which is required to make the most efficient use of the available bandwidth.

1) Joint Carrier and Power Allocation: In this section, we review the JPCA module [21], which is depicted in Fig. 3. The first block computes the channel gain matrices at a carrier level $\mathbf{G}(m)$ as described in Section III-B; this information is used in the second block to identify the FS receiver that gets the highest level of interference from the cognitive satellite system, i.e., for each $k$-th FSS user, $l_{\mathrm{w}}(m, k)=\max _{l}[\mathbf{G}(m)]_{k}$, where $[\mathbf{G}(m)]_{k}$ denotes the $k$-th row of matrix $\mathbf{G}(m)$ and $l_{\mathrm{w}}(m, k)$ 


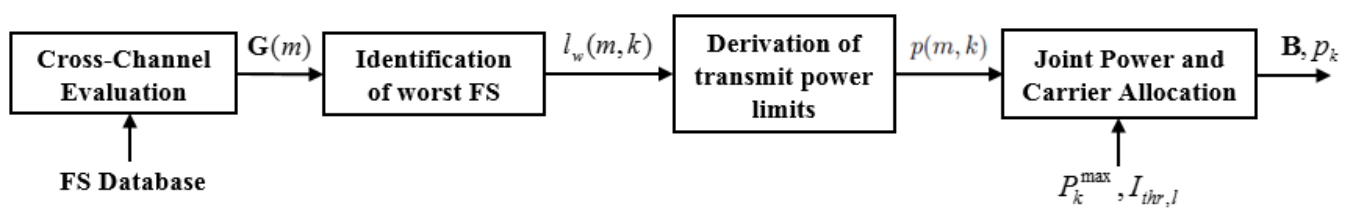

Fig. 3. Block diagram of the Joint Power and Carrier Allocation (JPCA) module.

indicates worst FS station in terms of interference of user $k$ operating in carrier $m$. These worst FS stations are used in the third block to determine the maximum allowable transmit power per user and per carrier. Let $I_{\mathrm{thr}, l_{\mathrm{w}}(m, k)}$ [W] denote the interference limit of the FS receiver that gets the highest interference level from the $k$-th RCST. This interference limit is divided among the total number of possible interferers, i.e., the number of RCSTs that fit within the FS receiver bandwidth. In mathematical notation,

$$
I_{\mathrm{w}}(m, k)=I_{\mathrm{thr}, l_{\mathrm{w}}(m, k)}\left(\frac{B^{\mathrm{FS}}}{B^{\mathrm{FSS}}}\right)^{-1} .
$$

Consequently, the power assignment should respect the interference constraint given by,

$$
I_{\mathrm{W}}(m, k) \geq p(m, k) \cdot G_{\mathrm{Tx}}^{F S S}\left(\theta_{k, l}\right) \cdot G_{\mathrm{Rx}}^{\mathrm{FS}}\left(\theta_{l, k}\right) \cdot L_{s} \cdot L_{d} .
$$

Solving (14), we obtain the following maximum transmission power that each RCSTs should not exceed to guarantee the incumbent FS system protection

$$
p_{\max }(m, k)=\frac{I_{\mathrm{w}}(m, k)}{G_{\mathrm{Tx}}^{F S S}\left(\theta_{k, l}\right) \cdot G_{\mathrm{Rx}}^{\mathrm{FS}}\left(\theta_{l, k}\right) \cdot L_{s} \cdot L_{d}} .
$$

Note that there could be some frequencies where no FS is deployed leading to $p_{\max }(m, k) \rightarrow \infty$ or very good conditions in which $p_{\max }(m, k)>P_{k}^{\max }$. Moreover, we might face the opposite situation where the interference constraint is too strong and the value of $p_{\max }(m, l)$ is below the minimum required power to close the link, namely $P_{m, k}^{\min }$. To overcome this infeasibility conditions, the resulting $p_{\max }(m, k)$ are subject to the following adjustments,

$$
p(m, k)= \begin{cases}P_{k}^{\max } & \text { if } p_{\max }(m, k)>P_{k}^{\max } \\ p_{\max }(m, k) & \text { if } P_{m, k}^{\min } \leq p_{\max }(m, k)<P_{k}^{\max } \\ 0 & \text { otherwise }\end{cases}
$$

The transmit powers given by (16) ensure that any combination of them never results in an aggregate interference above the acceptable threshold. Having solved the incumbent system protection, the next last block in Fig. 3 is devoted to optimally allocate carriers and powers so that the sum-rate of the satellite return link is maximized.

The objective is, thus, to design the carrier allocation matrix A introduced in Section IV-A2 but for the return link. Note that having obtained the carrier allocation matrix $\mathbf{A}$, it is straightforward to compute the corresponding power allocation as $p_{k}=\mathbf{a}_{k}^{H} \mathbf{p}_{k}$, where $\mathbf{p}_{k}=\left[\begin{array}{llll}p(1, k) & p(2, k) & \ldots & p(M, k)\end{array}\right]$. Therefore, solving (12) with the SINR values corresponding to the uplink scenario will provide a joint power and carrier

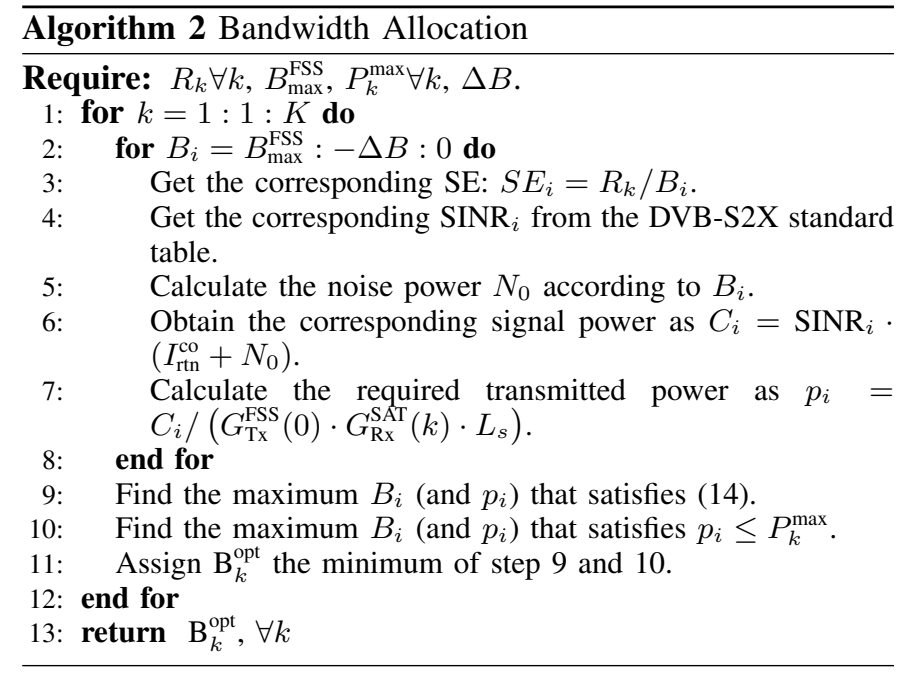

assignment. This is,

$$
\max _{\mathbf{A}}\left\|\operatorname{vec}\left(\mathbf{A} \odot \mathbf{R}\left(\mathbf{S I N R}_{\mathrm{rtn}}\right)\right)\right\|_{l_{1}} \quad \text { s.t. } \quad \sum_{k=1}^{K} \mathbf{a}_{k}(m)=1 .
$$

2) Bandwidth Allocation: With the previous JPCA, we assumed the user bandwidth to be fixed. Here, we consider the satellite bandwidth to be dynamically allocated to the RCSTs based on users' rate demands. This is more suitable for bursty traffic like the one supported in the satellite return link. As a result, higher spectrum efficiency is expected.

Let us assume that each RCST request a specific minimum rate, denoted $R_{k}$. Given an assigned bandwidth $B_{k}$ and assuming, this rate can be mapped to a particular Spectral Efficiency (SE) as follows, $S E_{k}=R_{k} / B_{k}$. Then, the minimum SINR value associated with $S E_{k}$ can be extracted from the DVBS2X standard tables [33]. Therefore, for each rate demand $R_{k}$, we have multiple minimum SINR values depending on the assigned bandwidth. To solve this problem, we proposed an ad-hoc algorithm to come up with the optimal bandwidth. The procedure is described in detail in Algorithm 2. The algorithm obtains the required transmitted power per different bandwidth values to achieve the requested demand. Then, it selects the minimum bandwidth that provides a transmit power below $P_{k}^{\max }$ and that satisfies the interference threshold at the terrestrial system.

\section{Simulation Results}

In this section, we evaluate the performance of the proposed cognitive exploitation techniques considering realistic system parameters. To do so, we select a country for each of the 

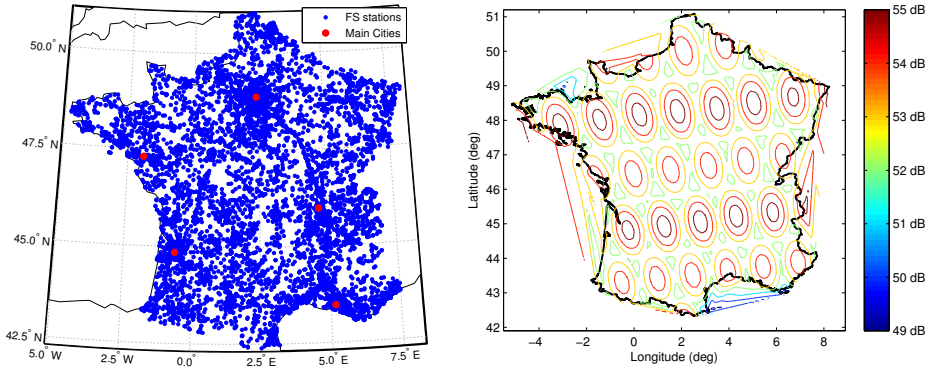

Fig. 4. (a) FS distribution map for France, and (b) Satellite multi-beam pattern covering France.
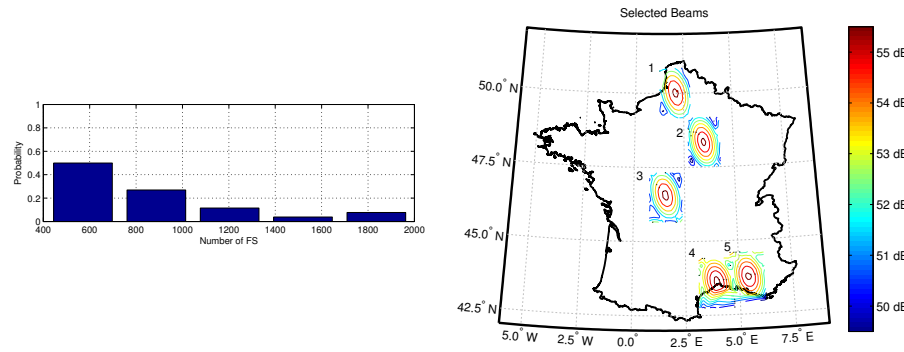

Fig. 5. (a) Pdf of the beams shown in Fig. 4(b) according to the number of FS links contained in each beam, and (b) Selected beams for downlink evaluation.

scenarios under evaluation, namely France for the cognitive satellite downlink and Finland for the uplink counterpart. The reason behind this choice are, firstly, these two countries are within the coverage of $13^{\circ} \mathrm{E}$ EUTELSAT satellite, whose multi-beam pattern and adjacent beam interference were obtained from Thales Alenia Space (TAS) in the framework of FP7 project CoRaSat [51]. Secondly, FS links databases for these countries are the most complete that we found.

The methodology to evaluate the cognitive satellite gain is the following. Each country under evaluation is covered by a number of satellite beams. From those beams, we choose the set of the most representative ones based on FS link deployment density and we carry out the throughput evaluation for each of them. The resulting throughput calculations are lastly combined with a proper weighting factor to give the final gain evaluation result. More details are provided in the following sections. Note that this section focuses on throughput calculations. Results related to inter-system interference can be found in [20], [21].

\section{A. Cognitive Satellite Downlink Evaluation}

The FS microwave links database related to France have been obtained from ITU-R BR International Frequency Information Circular (BR IFIC) database [52], which contains more than 12,000 entries with information listed on a station by station basis with the location of the antenna, maximum antenna gain, transmit power, channel bandwidth, etc. The geographical distribution of these FS links is illustrated in Fig. 4(a).

For the satellite modeling, we use the real multi-beam pattern covering France from the $13^{\circ} \mathrm{E}$ EUTELSAT satellite, which is shown in Fig. 4(b) (the reader should focus on the
TABLE I

DETAILED INFORMATION ABOUT THE SELECTED BEAMS

\begin{tabular}{|c|c|c|c|c|}
\hline Beam number & FS links & Weight & Latitude $^{*}$ & Longitude $^{*}$ \\
\hline 1 & 1681 & 0.077 & $50.0072^{\circ} \mathrm{N}$ & $2.0397^{\circ} \mathrm{E}$ \\
\hline 2 & 1522 & 0.039 & $48.3364^{\circ} \mathrm{N}$ & $3.5137^{\circ} \mathrm{E}$ \\
\hline 3 & 635 & 0.5 & $46.4896^{\circ} \mathrm{N}$ & $1.5707^{\circ} \mathrm{E}$ \\
\hline 4 & 906 & 0.269 & $43.5876^{\circ} \mathrm{N}$ & $3.9157^{\circ} \mathrm{E}$ \\
\hline 5 & 1220 & 0.115 & $43.7635^{\circ} \mathrm{N}$ & $5.3898^{\circ} \mathrm{E}$ \\
\hline
\end{tabular}

Location of the beam center

TABLE II

SIMULATION PARAMETERS FOR DOWNLINK

\begin{tabular}{|c|c|}
\hline Parameter & Value \\
\hline$B^{\mathrm{FSS}}$ & $62.4 \mathrm{MHz}$ \\
Shared band & $17.7-19.7 \mathrm{GHz}(32$ carriers $)$ \\
Exclusive band & $19.7-20.2 \mathrm{GHz}(8$ carriers $)$ \\
\hline Satellite location & $13^{\circ} \mathrm{E}$ \\
EIRP satellite & $65 \mathrm{dBW}$ \\
$G_{\mathrm{Tx}}^{\mathrm{SAT}}(k)$ & Between 49.60 and $54.63 \mathrm{dBi}$ \\
{$\left[C / I_{\text {fod }}^{\mathrm{co}}\right]$} & Between -7.37 and $-14.16 \mathrm{~dB}$ \\
Reuse pattern & 4 color $(\mathrm{freq} . / \mathrm{pol})$. \\
Satellite height & $35,786 \mathrm{Km}$ \\
$G_{\mathrm{Rx}}^{\mathrm{FSS}}(0)$ & $42.1 \mathrm{dBi}$ \\
$N_{0}$ & $-126.46 \mathrm{dBW}$ \\
Terminal height & $15 \mathrm{~m}$ \\
LNBs at the terminal & 3 \\
\hline
\end{tabular}

beams plotted inside the country boarders and ignore the lines in the sea area, which are useless). For the selection of the most representative beams, we computed the histogram to approximate the Probability Density Function (pdf) of these beams according to the number of FS links contained in each beam (in a $140 \mathrm{~km}$-radius area from the beam centre). Figure 5(a) shows the corresponding pdf. For the evaluation, we selected one beam per each pdf bar in Fig. 5(a) and the weight used for averaging the individual results was selected according to the corresponding probability extracted from Fig. 5(a). The selected beams are depicted in Fig. 5(b) and Table I provides detailed location and weighting factor of each one.

The performance of the cognitive satellite downlink was evaluated by averaging over 50 independent FSS terminal geographical distributions, which were selected uniformly at random for each realization within the considered beam footprint according to the population density database obtained from the NASA Socioeconomic Data and Applications Center (SEDAC) [53]. The number of FSS terminals $K$ is set to be 40, which coincides with the number of carriers to be assigned, i.e., $M$. Each FSS terminal is assumed to be equipped with 3 LNBs. A summary of the most relevant parameters and the FSS link budget details are presented in Table II.

For our analysis, we consider the following cases:

- Case 1 - Exclusive only: In this case, the SINRs and user rates are calculated using only exclusive carriers. This denotes the conventional system without the use of cognitive SatCom.

- Case 2 - Shared plus exclusive without FS interference: In this case, the SINRs and user rates were calculated considering both shared and exclusive carriers, but without considering the FS system. This represents the scenario where the additional spectrum is allocated exclusively to FSS system. This case does not exist in 
TABLE III

Throughrut Per Beam (MBPS) - Cognitive Satellite Downlink

\begin{tabular}{|c|c|c|c|c|c|c|c|c|}
\hline \multirow[t]{2}{*}{ Beam number } & \multirow[t]{2}{*}{ Weight } & \multicolumn{2}{|c|}{ Case 1} & \multicolumn{2}{|c|}{ Case 2} & \multicolumn{3}{|c|}{ Case 3} \\
\hline & & W/o CA & w/ CA & w/o CA & $\mathrm{w} / \mathrm{CA}$ & w/o CA & $\mathrm{w} / \mathrm{CA}$ & $\mathrm{w} / \mathrm{CA}+\mathrm{BF}$ \\
\hline 1 & 0.077 & 675.10 & 675.42 & 3414.17 & 3419.78 & 3413.73 & 3419.78 & 3468.05 \\
\hline 2 & 0.039 & 679.13 & 679.4 & 3404.98 & 3410. & 3404.25 & 3410 & 7.66 \\
\hline 3 & 0.5 & 660.42 & 660.72 & 3304.87 & 3309.07 & 3304.11 & 3309.08 & 3331.52 \\
\hline 4 & 0.269 & 725.76 & 725.95 & 3641.67 & 3646.2 & 3640.1 & & 1.03 \\
\hline 5 & 0.115 & 718.47 & 718.85 & 3626.94 & 3646.07 & 3623.62 & 3645.79 & 3659.71 \\
\hline Per beam average th & 1 & 686.56 & 686.84 & 3444.97 & 3451.16 & 3443.74 & 3451.13 & 3473.46 \\
\hline atellite total throug & & 171.64 & 171.71 & 861.24 & 862.79 & 860.94 & 862.78 & 868.37 \\
\hline
\end{tabular}
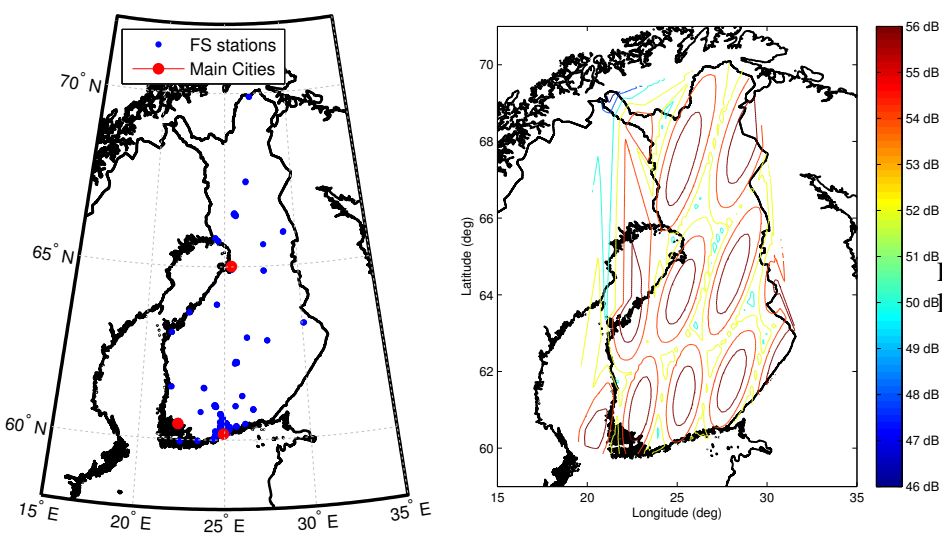

Fig. 6. (a) FS distribution map for Finland, and (b) Satellite multi-beam pattern covering Finland.

practice but is considered for comparison purposes.

- Case 3 - Shared plus exclusive with FS interference: In this case, the SINRs and user rates were calculated considering both shared and exclusive carriers, and considering the FS system. This depicts the scenario where FSS system shares the band primarily allocated to the FS systems.

The results of the evaluated beams are shown in Table III. The SINR threshold which determines the application of BF is considered to be $\mathrm{SINR}_{t h}=9.71 \mathrm{~dB}$, which corresponds to the 16APSK 13/18 ModCod based on the DVB-S2X specifications [33]. The case indicated as "w/o CA" means that a random carrier allocation has been carried out, which is worse or as good as the optimal CA case indicated with "w/ CA".

From Table III, it can be noted that the throughput values significantly differ across the considered beams even for the case of exclusive only case, which is due to different beam gains $G_{\mathrm{Tx}}^{\mathrm{SAT}}(k)$ and adjacent beam interference $\left[C / I_{\mathrm{fwd}}^{\mathrm{co}}\right]$ values over these beams.

In Table III, we first calculate the per beam average throughput considering the weighting factors, and secondly, the total system throughput was obtained for each individual case under study. It can be observed that the application of CA in the exclusive only case does not provide much benefit. This is because all SINR values are quite good and there is not enough room for improvement. The per beam average throughput shown in Table III makes evident the gains achieved when using the $2 \mathrm{GHz}$ of extra spectrum on top of the traditional $500 \mathrm{MHz}$ of the exclusive band. Even without considering the smart resource allocation strategy, the FSS system increases its overall throughput from 686.56 to

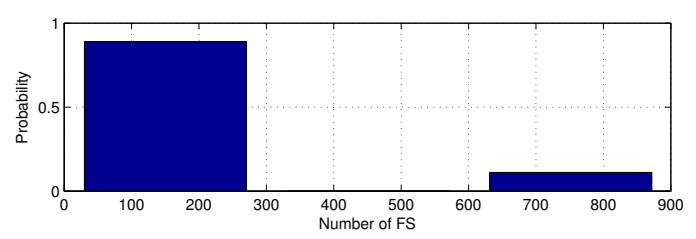

Fig. 7. Pdf of the beams shown in Fig. 6(b) according to the number of FS links contained in each beam.

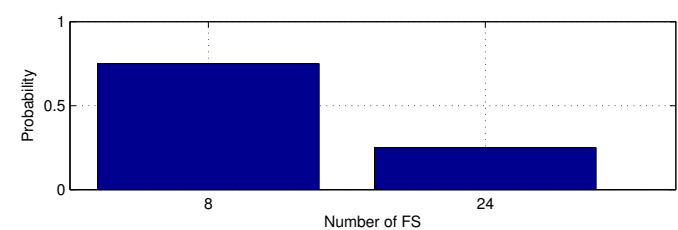

Fig. 8. Pdf of the beams shown in Fig. 6(b) according to the number of FS links contained in each beam, excluding the Helsinki area.

3443.74 Mbps by accommodating some FSS terminals into the shared band, which can be increased up to $3473.46 \mathrm{Mbps}$ when considering the proposed $\mathrm{CA}$ and BF techniques. In particular, the average throughput per beam gain obtained with the proposed CA technique is $402.67 \%$ over the exclusive only case, which can be further increased with a $3.25 \%$ using $\mathrm{BF}$ technique.

It is worth mentioning that the proposed cognitive exploitation framework can achieve similar average throughput as if there were no FS system present in the scene.

At the very end of Table III, the total satellite throughput was calculated considering the total 250 beams of the satellite providing coverage to the whole Europe. Clearly, the use of resource allocation techniques in this scenario provides overall satellite throughput of the order of $870 \mathrm{Gbps}$, which is close to the next generation High Throughput Satellite (HTS) terabit system requirements [5].

\section{B. Cognitive Satellite Uplink Evaluation}

The FS database in the case of Finland was obtained from the FInnish COmmunications Regulatory Authority (FICORA). This database contains 984 entries for the $28 \mathrm{GHz}$ band. The geographical distribution of the FS stations can be found in Fig. 6(a).

The nominal interference threshold for an FS receiver was based on the long term interference which is set at -146 $\mathrm{dBW} / \mathrm{MHz}$.

For the satellite beam pattern, we use the same $13^{\circ} \mathrm{E}$ EUTELSAT satellite, whose footprint over Finland is shown 
TABLE IV

DETAILED INFORMATION ABOUT THE SELECTED BEAMS

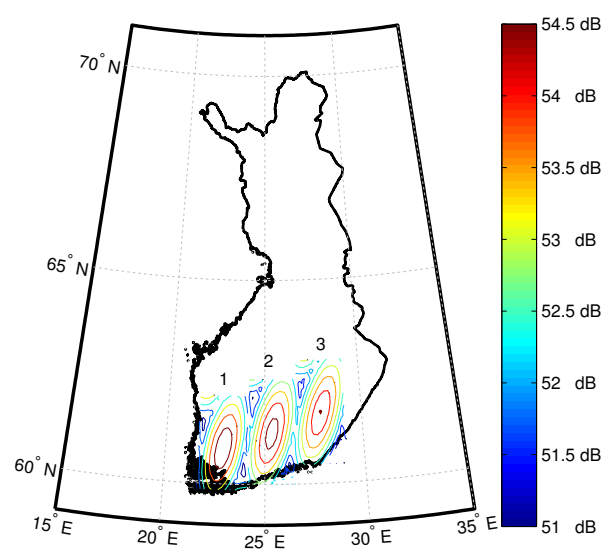

Fig. 9. Selected beams for uplink evaluation.

in Fig. 6(b). Again, for the selection of the most representative beams, we computed the histogram to approximate the pdf of these beams according to the number of FS links contained in a $140 \mathrm{Km}$-radius area from the center of each beam. Figure 7 shows the corresponding pdf computed with 3 bars (although the second bar is empty). From Fig. 7, one can observe that there are beams with high density of FS links and others with very sparse distribution of FS links in Finland. We selected two beams from the first bar and one beam for the third bar in Fig. 7. The beam corresponding to the third bar in Fig. 7 is the beam giving coverage to the Helsinki area. The weight of this beam will be 0.111 , corresponding to its probability. In order to select two beams from the first bar in Fig. 7, we reconstruct the pdf but excluding the Helsinki beam. The second pdf is depicted in Fig. 8, from which we select one beam per each pdf bar. Taking into account both pdfs (Fig. 7 and Fig. 8) we derived the corresponding weights. The selected beams are depicted in Fig. 9 and Table IV provides detailed location and weighting factor of each one.

As we did for the downlink evaluation, the uplink performance was evaluated by averaging over 50 independent FSS terminal geographical distributions, which were selected uniformly at random for each realization within the considered beam footprint according to the population density database obtained from [53]. In this case, the number of FSS terminals $K$ is set to be 356, which coincides with the number of carriers to be assigned, i.e., $M$. A summary of the most relevant parameters and the FSS link budget details are presented in Table V.

The results of the JPCA in the three evaluated beams are shown in Table VI using the same format as the one used for the cognitive downlink evaluation. It is important to keep in mind that, in this scenario, when using the exclusive only spectrum band (Case 1) and the shared plus exclusive in the absence of FS links (Case 2), the RCST transmit power is fixed to the maximum, i.e., $P_{k}^{\max }=7.9 \mathrm{dBW}, \forall k$. Note that the proposed JPCA ensures that any combination of powers never results in an aggregate interference above the acceptable threshold. Therefore, when using the shared plus exclusive in

\begin{tabular}{|c|c|c|c|c|}
\hline Beam number & FS links & Weight & Latitude $^{*}$ & Longitude $^{*}$ \\
\hline 1 & 32 & 0.222 & $60.8801^{\circ} \mathrm{N}$ & $22.8749^{\circ} \mathrm{E}$ \\
\hline 2 & 902 & 0.111 & $61.2905^{\circ} \mathrm{N}$ & $25.4073^{\circ} \mathrm{E}$ \\
\hline 3 & 6 & 0.667 & $61.7522^{\circ} \mathrm{N}$ & $27.878^{\circ} \mathrm{E}$ \\
\hline
\end{tabular}

Location of the beam center

TABLE V

SimUlation PARAMETERS FOR UPLINK

\begin{tabular}{|c|c|}
\hline Parameter & Value \\
\hline$B^{\mathrm{FS}}$ & $7 \mathrm{MHz}$ \\
Shared band & $27.5-29.5 \mathrm{GHz}(285$ carriers $)$ \\
Exclusive band & $29.5-30 \mathrm{GHz}(71$ carriers) \\
\hline Parameters for FSS system & $13^{\circ} \mathrm{E}$ \\
\hline Satellite location & $50 \mathrm{dBW}$ \\
EIRP FSS terminal & Between 44.43 and $57.88 \mathrm{dBi}$ \\
$G_{\mathrm{Rx}}^{\text {SAT }}(k)$ & Between 5.80 and $20.74 \mathrm{~dB}$ \\
{$\left[C / I_{\mathrm{rtn}}^{\text {co }}\right]$} & 4 color $($ freq./pol. $)$ \\
Reuse pattern & $35,786 \mathrm{Km}$ \\
Satellite height & $42.1 \mathrm{dBi}$ \\
$G_{\mathrm{Tx}}^{\mathrm{FSS}}(0)$ & $-131.78 \mathrm{dBW}$ \\
$N_{0}$ & $15 \mathrm{~m}$ \\
\hline
\end{tabular}

the presence of FS links (Case 3), we refer to "w/o JPCA" when a random carrier allocation has been carried out but satisfying interference constraints. The later should be worse or as good as the optimal JPCA case indicated with "w/ JPCA".

The main conclusion that can be extracted from Table VI is that the additional spectrum together with the optimal JPCA module provides $398.12 \%$ improvement over the conventional exclusive band $(29.5-30 \mathrm{GHz})$ case. It can be observed that the optimal JPCA provides a gain of $0.8 \%$ over the suboptimal JPCA. It is important to highlight that there is only one beam in Finland with high deployment of FS links, and thus, with strong interference restriction requirements. The main conclusion is that using the proposed JPCA, we can achieve the same throughput as if there were no FS system, while ensuring protection to them. Extending the usable Ka band spectrum together with the proposed resource allocation techniques in this scenario provides overall system throughput of the order of $1380 \mathrm{Gbps}$. The FS microwave deployment in the $28 \mathrm{GHz}$ is rather sparse compared to the $18 \mathrm{GHz}$ counterpart, which justifies the higher gains achieved in the cognitive uplink compared to the cognitive downlink.

Regarding the bandwidth allocation technique, we randomly generate rate demands per user and per realization. We assume that these demands are not greater than the maximum that can be achieved with $7 \mathrm{MHz}$ bandwidth. The results obtained with the proposed technique are shown in Table VII. The mean user demand is half of the maximum that was assigned with JPCA and the 7MHz bandwidth and, therefore, bandwidth per user is reduced as well as transmitted power. The proposed bandwidth allocation scheme satisfies the user rate demands and prevents an unnecessary waste of resources by maximizing the effectiveness of their utilization. 
TABLE VI

ThroughPut PER BEAM (MBPS) - Cognitive SATELlite UPLinK

\begin{tabular}{|c|c|c|c|c|c|c|c|}
\hline \multirow[t]{2}{*}{ Beam number } & \multirow[t]{2}{*}{ Weight } & \multicolumn{2}{|c|}{ Case 1 } & \multicolumn{2}{|c|}{ Case 2} & \multicolumn{2}{|c|}{ Case 3} \\
\hline & & w/o CA & $\mathrm{w} / \mathrm{CA}$ & w/o CA & $\mathrm{w} / \mathrm{CA}$ & w/ JPCA (subopt) & $\mathrm{w} / \mathrm{JPCA}(\mathrm{opt})$ \\
\hline$\frac{1}{2}$ & $\begin{array}{l}0.222 \\
0.111\end{array}$ & $\begin{array}{l}1098.79 \\
1015.55\end{array}$ & $\begin{array}{l}1098.79 \\
1015.55\end{array}$ & $\begin{array}{l}5467.67 \\
5077.65\end{array}$ & $\begin{array}{l}5474.58 \\
5081.19\end{array}$ & $\begin{array}{l}5453.37 \\
4765.63\end{array}$ & $\begin{array}{l}5474.58 \\
5081.19\end{array}$ \\
\hline 3 & 0.667 & 1127.58 & 1127.58 & 5607.49 & 5612.45 & 5606.72 & 5612.45 \\
\hline Per beam average throughput & 1 & 1108.75 & 1108.75 & 5517.64 & 5522.87 & 5479.32 & 5522.87 \\
\hline Satellite total throughput (considering 250 & & 277.2 & 277.2 & 1379.4 & 1380.7 & 1369.8 & 1380.7 \\
\hline
\end{tabular}

TABLE VII

BANDWIDTH ALLOCATION RESULTS*

\begin{tabular}{|c|c|c|c|}
\hline Beam number & Mean Rate [Mbps] & Mean Bandwidth [MHz & Mean Power [dBW \\
\hline 1 & $7.7479(15.329)$ & $3.501(7)$ & $3.4472(7.8999)$ \\
\hline 2 & $7.0504(14.227)$ & $3.4429(7)$ & $2.5154(7.864)$ \\
\hline 3 & $7.7213(15.715)$ & $3.3949(7)$ & $3.7103(7.9)$ \\
\hline
\end{tabular}

*Values in parenthesis represent the values values achieved with JPCA with $7 \mathrm{MHz}$ bandwidth.

\section{Vi. Conclusions}

In this paper, we proposed a novel spectrum exploitation framework for enabling the operation of cognitive FSS terminals in the band $17.7-19.7 \mathrm{GHz}$ for the satellite downlink and $27.5-29.5 \mathrm{GHz}$ for the satellite uplink. A thorough system gain evaluation exercise has been performed on the basis of available deployment data for the cognitive satellite downlink and uplink. The numerical results presented in this paper showed that the combination of the exclusive Ka-band frequency assignment with the non-exclusive frequency bands that are primarily assigned to FS microwave links provides significant throughput gains to the FSS system.

For the cognitive satellite downlink scenario, the average throughput per beam gain obtained with the proposed CA technique is $402.67 \%$ over the conventional exclusive case, which can be further increased with a $3.25 \%$ if we assume beamforming capabilities at the FSS terminal.

For the cognitive satellite uplink scenario, the gain achieved using the proposed JPCA and the non-exclusive bands is around $394.19 \%$ compared to the conventional non-cognitive satellite uplink, which can be increased with $3.93 \%$ when the powers and carriers are optimally assigned. Moreover, we proposed a bandwidth allocation scheme based on users' rate requests which prevents an unnecessary waste of spectrum while satisfying users' demands. Note that, in all cases, the cognitive satellite system is guaranteed to never exceed the prescribed interference threshold.

The gain achieved with the proposed resource allocation techniques will surely increase with the advent of $5 \mathrm{G}$ mobile wireless technology and the expected densification of the mmWave terrestrial backhauling network. Also, future satellite traffic demands indicate that more bandwidth (especially on the uplink) will be required and thus, the proposed resource allocation techniques become important mechanisms to address the interference issue in cognitive satellite communications.

There are other scenarios in which the proposed cognitive spectrum exploitation techniques can be adopted, such as considering cognitive Earth Stations on Mobile Platforms (ESOMPs) operating in the considered bands or considering Non-Geostationary (NGEO) satellite communication systems operating in the shared bands. The proposed methods can be applied in these applications, which are regarded as our potential directions of future work.

\section{REFERENCES}

[1] W. Wu, "Satellite Communications," Proc. IEEE, vol. 85, no. 6, pp. 998-1010, Jun, 1997.

[2] B. Evans, Satellite Communication Systems. IET Telecommunications Series, London, UK, Dec, 1999.

[3] P. Eskelinen, "Satellite Communications Fundamentals [Book Review]," IEEE Aerospace and Electronic Systems Magazine, vol. 16, no. 10, pp. 22-23, Oct, 2001.

[4] T. D. Cola, D. Tarchi, and A. Vanelli-Coralli, "Future Trends in Broadband Satellite Communications: Information Centric Networks and Enabling Technologies," International Journal of Satellite Communications and Networking, Apr, 2015.

[5] A. Kyrgiazos, B. Evans, P. Thompson, P. Mathiopoulos, and S. Papaharalabos, "A Terabit/Second Satellite System for European Broadband Access: A Feasibility Study," Int. J. Satell. Commun. Network., vol. 32, no. 2, pp. 63-92, Mar, 2014.

[6] O. Vidal, G. Verelst, J. Lacan, E. Alberty, J. Radzik, and M. Bousquet, "Next Generation High Throughput Satellite System," IEEE AESS European Conf. on Satellite Telecommunications (ESTEL), Rome, Italy, Oct, 2012.

[7] D. Brandel, W. Watson, and A. Weinberg, "NASA's Advanced Tracking and Data Relay Satellite System for the Years 2000 and Beyond," Proceedings of the IEEE, vol. 78, no. 7, pp. 1141-1151, Jul, 1990.

[8] D. Christopoulos, S. Chatzinotas, and B. Ottersten, "Multicast multigroup precoding and user scheduling for frame-based satellite communications," IEEE Trans. Wireless Communications, vol. PP, no. 99, pp. $1-1,2015$.

[9] A. Vanelli-Coralli, A. Guidotti, D. Tarchi, S. Chatzinotas, S. Maleki, S. Sharma, N. Chuberre, B. Evans, M. Lopez-Benitez, W. Tang, J. Grotz, and K. Liolis, Cooperative and Cognitive Satellite Systems. Oxford, UK: Academic Press, 2015, ch. Cognitive Radio Scenarios for Satellite Communications: The CoRaSat Project.

[10] S. Sharma, S. Chatzinotas, and B. Ottersten, "Cognitive Beamhopping for Spectral Coexistence of Multibeam Satellites," Int. J. Satellite Commun. and Networking, Mar, 2014.

[11] M. Hoyhtya, J. Kyrolainen, A. Hulkkonen, J. Ylitalo, and A. Roivainen, "Application of Cognitive Radio Techniques to Satellite Communication," IEEE Symp. on Dynamic Spectrum Access Networks (DySPAN), Bellevue, WA, USA, Oct, 2012.

[12] S. Maleki, S. Chatzinotas, B. Evans, K. Liolis, J. Grotz, A. VanelliCoralli, and N. Chuberre, "Cognitive Spectrum Utilization in Ka Band Multibeam Satellite Communications," IEEE Communication Magazine, vol. 53, no. 3, pp. 24-29, Mar, 2015.

[13] S. Haykin, "Cognitive radio: brain-empowered wireless communications," IEEE Journal on Selected Areas in Communications, vol. 23, no. 2, pp. 201-220, 2005. 
[14] V. Icolari, A. Guidotti, D. Tarchi, and A. Vanelli-Coralli, "Interference Estimation in Satellite Cognitive Radio Systems," IEEE Int. Conf. on Communications (ICC), London, UK, Jun, 2015.

[15] D. Tarchi, V. Icolari, J. Grotz, A. Vanelli-Coralli, and A. Guidotti, "On the feasibility of Interference Estimation Techniques in Cognitive Satellite Environments with Impairments," submitted to Int. Conf. Wireless and Satellite Systems (WiSats), Bradford, UK, Jul, 2015.

[16] B. Evans, P. Thompson, E. Lagunas, S. Sharma, D. Tarchi, and V. Icolari, "Extending the Usable Ka band Spectrum for Satellite Communications: The CoRaSat Project," submitted to Int. Conf. Wireless and Satellite Systems (WiSats), Bradford, UK, Jul, 2015.

[17] P. Thompson and B. Evans, "Analysis of Interference between Terrestrial and Satellite Systems in the Band 17.7 to $19.7 \mathrm{GHz}$," Workshop on Cognitive Radios and Networks for Spectrum Coexistence of Satellite and Terrestrial Systems, IEEE Int. Conf. on Communications (ICC), London, UK, Jun, 2015.

[18] S. Maleki, S. Chatzinotas, J. Krause, K. Liolis, and B. Ottersten, "Cognitive Zone for Broadband Satellite Communication in 17.3-17.7 GHz Band," IEEE Wireless Communication Letters, vol. PP, no. 99, pp. 1-1, Mar, 2015.

[19] A. Goldsmith, S. A. Jafar, I. Maric, and S. Srinivasa, "Breaking Spectrum Gridlock with Cognitive Radio: An Information Theoretic Perspective," Proceedings of the IEEE, vol. 97, no. 5, pp. 894-914, May, 2009.

[20] S. Sharma, E. Lagunas, S. Maleki, S. Chatzinotas, J. Grotz, J. Krause, and B. Ottersten, "Resource Allocation for Cognitive Satellite Communications in Ka-band (17.7-19.7 GHz)," Workshop on Cognitive Radios and Networks for Spectrum Coexistence of Satellite and Terrestrial Systems, IEEE Int. Conf. on Communications (ICC), London, UK, Jun, 2015.

[21] E. Lagunas, S. Sharma, S. Maleki, S. Chatzinotas, J. Grotz, J. Krause, and B. Ottersten, "Resource Allocation for Cognitive Satellite Uplink and Fixed-Service Terrestrial Coexistence in Ka-band," Int. Conf. on Cognitive Radio Oriented Wireless Networks (CROWNCOM), Doha, Qatar, Apr, 2015.

[22] A. Guidotti, V. Icolari, D. Tarchi, A. Vanelli-Coralli, S. Sharma, E. Lagunas, S. Maleki, S. Chatzinotas, J. Grotz, J. Krause, E. Corbel, B. Evans, and P. Thompson, "Spectrum Awareness and Exploitation for Cognitive Radio Satellite Communications," European Conference on Networks and Communications (EUCnC), Paris, France, Jun, 2015.

[23] D2.3, "Scenarios Definition and Selection," CoRaSat FP7 project, Oct, 2012, http://www.ict-corasat.eu/documents/deliverables.

[24] ECC/DEC/(00)07, "The shared use of the band 17.7-19.7 GHz by the fixed service and Earth stations of the fixed-satellite service (space-toEarth) ," Electronic Communication Committee, Oct, 2000.

[25] S. Sharma, S. Maleki, S. Chatzinotas, J. Grotz, and B. Ottersten, "Implementation Issues of Cognitive Radio techniques for Ka-band (17.719.7 GHz) SatComs," Advanced Satellite Multimedia Systems (ASMS) Conference and Signal Processing for Space Communications (SPSC) Workshop, Livorno, Italy, Sep, 2014.

[26] J. Lei and M. Vazquez-Castro, "Joint Power and Carrier Allocation for the Multibeam Satellite Downlink with Individual SINR Constraints," IEEE Int. Conf. on Communications (ICC), Cape Town, South Africa, May, 2010.

[27] J. Choi and V. Chan, "Optimum Power and Beam Allocation based on Traffic Demands and Channel Conditions Over Satellite Downlinks," IEEE Trans. on Wireless Communications, vol. 4, no. 6, pp. 2983-2993, Nov, 2005.

[28] L. Westbrook, T. Calladene, I. Berry, and N. Briscombe, "Carrier frequency allocation in FDMA military SATCOMs," Military Communications Conference (MILCOM), McLean, Virginia, USA, Oct, 2001.

[29] N. Celandroni, F. Davoli, E. Ferro, and A. Gotta, "Networking with Multi-Service GEO Satellites: Cross-Layer Approaches for Bandwidth Allocation," Journal of Satellite Communications and Networking, Special Issue on Cross Layer Protocols for Satellite Communication Networks: Part I, vol. 24, no. 5, pp. 387-403, Sep, 2006.

[30] V. Icolari, D. Tarchi, A. Guidotti, and A. Vanelli-Coralli, "Beam Pattern Allocation Strategies for Satellite Cognitive Radio Systems," Workshop on Cognitive Radios and Networks for Spectrum Coexistence of Satellite and Terrestrial Systems, IEEE Int. Conf. on Communications (ICC), London, UK, Jun, 2015.
[31] S. Sharma, S. Maleki, S. Chatzinotas, J. Grotz, J. Krause, and B. Ottersten, "Joint Carrier Allocation and Beamforming for Cognitive SatComs in Ka-band (17.3-18.1 GHz)," IEEE Int. Conf. on Communications (ICC), London, UK, Jun, 2015.

[32] W. Tang, P. Thompson, and B. Evans, "Frequency Sharing between, Satellite and Terrestrial Systems in the Ka Band: A Database Approach," IEEE Int. Conf. on Communications (ICC), London, UK, Jun, 2015.

[33] Digital Video Broadcasting (DVB), "DVB-S2X Standard," https://www. dvb.org/standards/dvb-s2x, [Online; accessed 14-Apr-2015].

[34] J. Grotz, B. Ottersten, and J. Krause, "Signal Detection and Synchronization for Interference Overloaded Satellite Broadcast Reception," IEEE Trans. Wireless Commun., vol. 9, no. 10, pp. 3052-3062, Oct, 2010.

[35] ECC/DEC/(05)01, "The use of the band $27.5-29.5 \mathrm{GHz}$ by the Fixed Service and uncoordinated Earth stations of the Fixed-Satellite Service (Earth-to-space)," Electronic Communication Committee, Mar, 2005.

[36] ITU-R F.758, "System parameters and considerations in the development of criteria for sharing or compatibility between digital fixed wireless systems in the fixed service and systems in other services and other sources of interference," Series of ITU-R Recommendations, Mar, 2012.

[37] ECC Report 184, "The Use of Earth Stations on Mobile Platforms Operating with GSO Satellite Networks in the Frequency Range 17.320.2 GHz and 27.5-30.0 GHz," Electronic Communication Committee, 2013.

[38] Digital Video Broadcasting (DVB), "DVB-RCS Standard," https://www.dvb.org/standards/dvb-rcs, [Online; accessed 14-Apr2015].

[39] D. Petraki, M. Anastasopoulos, and P. Cottis, "Dynamic Resource Allocation for DVB-RCS Networks," International Journal of Satellite Communications and Networking, vol. 26, no. 3, pp. 189-210, Jun, 2008.

[40] P. Chini, G. Giambene, D. Bartolini, M. Luglio, and C. Roseti, "Dynamic Resource Allocation based on a TCP-MAC Cross-Layer Approach for DVB-RCS Satellite Networks," International Journal of Satellite Communications and Networking, vol. 24, no. 5, pp. 367-385, Oct, 2006.

[41] A. Morell, G. Seco-Granados, and M. Vazquez-Castro, "Cross-Layer Design of Dynamic Bandwidth Allocation in DVB-RCS," IEEE Systems Journal, vol. 2, no. 1, pp. 62-73, Mar, 2008.

[42] A. Mohamed, M. Lopez-Benitez, and B. Evans, "Ka Band Satellite Terrestrial Co-Existence: A Statistical Modelling Approach," $K a$ and Broadband Communications, Navigation and Earth Observation Conf., Salerno, Italy, Oct, 2014.

[43] S. Vassaki, M. Poulakis, and A. Panagopoulos, "Optimal iSINR-based Power Control for Cognitive Satellite Terrestrial Networks," Trans. on Emerging Telecommunications Technologies, May, 2015.

[44] D3.3, "Adaptation and Design of Cognitive Techniques for Satellite Communications," CoRaSat FP7 project, Oct, 2014, http://www. ict-corasat.eu/documents/deliverables.

[45] ITU-R S.465-6, "Reference radiation pattern for earth station antennas in the fixed-satellite service for use in coordination and interference assessment in the frequency range from 2 to $31 \mathrm{GHz}$,' Series of ITU-R Recommendations, Jan, 2010.

[46] ITU-R F.1245-2, "Mathematical model of average and related radiation patterns for line-of-sight point-to-point fixed wireless system antennas for use in certain coordination studies and interference assessment in the frequency range from $1 \mathrm{GHz}$ to about $70 \mathrm{GHz}$," Series of ITU-R Recommendations, Mar, 2012.

[47] ITU-R P.526-13, "Propagation by diffraction," Series of ITU-R Recommendations, Nov, 2013.

[48] ECC/DEC/(06)03, "Exemption from Individual Licensing of high e.i.r.p. satellite terminals (HEST) operating within the frequency bands 10.7012.75 GHz or $19.70-20.20 \mathrm{GHz}$ space-to-Earth and $14.00-14.25 \mathrm{GHz}$ or 29.50-30.00 GHz Earth-to-space," Electronic Communication Committee, 2006.

[49] O. Frost, "An Algorithm for Linearly Constrained Adaptive Array Processing," Proc. IEEE, vol. 60, no. 8, pp. 926-935, Aug, 1972.

[50] H. Kuhn, "The Hungarian Method for the Assignment Problem," Naval Research Logistics Quarterly, vol. 2, pp. 83-97, 1955.

[51] European Commission FP7, "COgnitive RAdio for SATellite Communications - CoRaSat," http://www.ict-corasat.eu/.

[52] ITU, "BRIFIC for Terrestrial Services," http://www.itu.int/en/ITU-R/ terrestrial/brific, [Online; accessed 7-Oct-2014].

[53] NASA, "Socioeconomic Data and Applications Center (SEDAC)," http: //sedac.ciesin.columbia.edu, [Online; accessed 30-Apr-2015]. 
Eva Lagunas (S'09-M'13) received the M.Sc. and $\mathrm{Ph} . \mathrm{D}$. degrees in telecommunications engineering from the Polytechnic University of Catalonia (UPC), Barcelona, Spain, in 2010 and 2014, respectively. She was Research Assistant within the Department of Signal Theory and Communications, UPC, from 2009 to 2013. During the summer of 2009 she was a guest research assistant within the Department of Information Engineering, Pisa, Italy, developing TOA estimation algorithms in UWB systems. From November 2011 to May 2012 she held a visiting research appointment at the Center for Advanced Communications (CAC), Villanova University, PA, USA, where she worked on through-wall radar imaging technology. In September 2014, she joined the Interdisciplinary Centre for Security, Reliability and Trust (SnT), University of Luxembourg, as a Research Associate, where she works on hybrid terrestrial-satellite communications. Dr. Lagunas is an associate editor of EURASIP Journal on Advances in Signal Processing (JASP) since 2015.

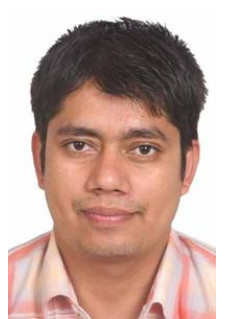

Shree Krishna Sharma (B.E., M.Sc. Engg., M.A., M. Res., Ph.D.) received the B.E. degree in electronics and communication from Birla Institute of Technology, Mesra, India; the M.Sc. degree in information and communication engineering from the Institute of Engineering, Pulchowk, Nepal; the M.A. degree in economics from Tribhuvan University Patan Multiple Campus, Patan, Nepal; the M.Res. degree in computing science from Staffordshire University, Staffordshire, U.K., and the Ph.D. degree from University of Luxembourg, Luxembourg in 2014. He is currently working as a Research Associate in Interdisciplinary Centre for Security, Reliability and Trust, University of Luxembourg, Luxembourg City, Luxembourg. In the past, he was also involved with Kathmandu University, Dhulikhel, Nepal, as a Teaching Assistant, and he served as a Part-Time Lecturer for eight engineering colleges in Nepal. He was with Nepal Telecom for more than four years as a Telecom Engineer in the field of information technology and telecommunication. He is the author of more than 30 technical papers in refereed international journals, scientific books, and conferences. He received an Indian Embassy Scholarship for his B.E. study, an Erasmus Mundus Scholarship for his M.Res. study, and an Aids Training-Research $\mathrm{Ph} . \mathrm{D}$. grant from the National Research Fund of Luxembourg. His research interests include cognitive wireless communications, satellite communications, and interference mitigation in heterogeneous wireless networks.

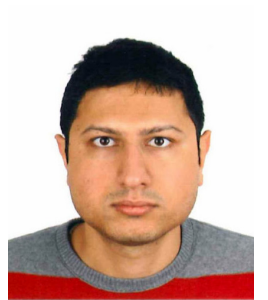

Sina Maleki received his $\mathrm{PhD}$ degree from Delft University of Technology, Delft, The Netherlands, in 2013, and his MSc from the same university in 2009. From July 2008 to April 2009, he was an intern student at the Philips Research Center, Eindhoven, The Netherlands, working on spectrum sensing for cognitive radio networks. Since August 2013, he has been working at the Interdisciplinary Centre for Security, Reliability and Trust, University of Luxembourg, where he is working on cognitive radio for satellite communications within the EU FP7 CoRaSat project, as well as Luxembourgish national projects CO2SAT, SeMIGod, and SATSENT. 


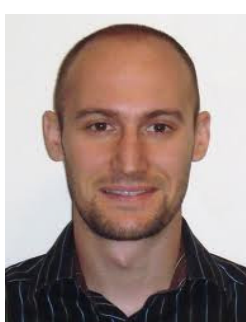

Symeon Chatzinotas is a Research Scientist at the Interdisciplinary Centre for Security, Reliability and Trust, University of Luxembourg. He has worked on numerous R\&D projects and has authored more than 120 technical papers in refereed international journals, conferences and scientific books. He has served as Technical Program Committee member in numerous conferences and he is a Senior IEEE member His research interests are in multiuser information theory, cooperative/cognitive communications, and wireless network optimization. Dr. Chatzinotas is a recipient of the 2014 Distinguished Contributions to Satellite Communications Award, Satellite and Space Communications Technical Committee, IEEE Communications Society.

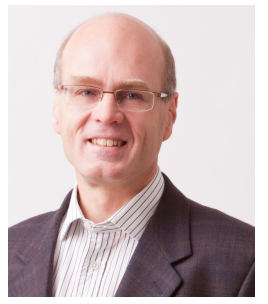

Björn Ottersten is Director for the Interdisciplinary Centre for Security, Reliability and Trust at the University of Luxembourg. As Digital Champion of Luxembourg, he acts as an adviser to the European Commissioner. He has held high level research and teaching positions at various universities, and was Director of Research at ArrayComm Inc, a startup in San Jose, CA, based on his own patented technology. He has authored over 450 scientific papers, including many award-winning journal and conference papers. He is currently editor in chief of EURASIP Signal Processing Journal. Dr. Ottersten is a Fellow of the IEEE and EURASIP. In 2011 he received the IEEE Signal Processing Society Technical Achievement Award. His research interests include security and trust, reliable wireless communications, and statistical signal processing. Prof. Ottersten is a recipient of the 2014 Distinguished Contributions to Satellite Communications Award, Satellite and Space Communications Technical Committee, IEEE Communications Society. 